\title{
Two-hole assisted phaco-chop technique: a more efficient method for safe nucleofractis vertical chopping
}

\author{
Naomi Miyamoto $\cdot$ Shoko Kiritoshi $\cdot$ Rinko Akamime $\cdot$ Masayuki Akimoto
}

Received: 29 May 2020/ Accepted: 19 April 2021/Published online: 4 May 2021

(C) The Author(s) 2021

\begin{abstract}
Purpose To evaluate the use of the two-hole technique in augmenting the efficiency of surgeons-intraining when performing the phaco-chop technique. We hypothesized that drilling two holes in opposite angles to each other adjacent to the capsulorhexis would mimic a new lens equator. The phaco-tip and the chopper can be inserted into these holes at appropriate depths and may sandwich and fracture the lens more easily than conventional methods.

Methods The two-hole technique described above was performed by three first-year surgeons before they performed the standard phaco-chop technique. We collected data of their first 8 cases and analyzed a total of 64 cases: 16, divide-and-conquer; 24, two-hole method; 24, phaco-chop. The main outcome measures included the cumulative dissipated energy (CDE) and case ultrasound time (UST) with different phacoemulsification techniques.
\end{abstract}

Results The young practicing surgeons eventually performed the standard phaco-chop more safely after

Supplementary Information The online version contains supplementary material available at https://doi.org/10.1007/ s10792-021-01872-4.

N. Miyamoto $\cdot$ S. Kiritoshi $\cdot$ R. Akamime

M. Akimoto $(\square)$

Department of Ophthalmology, Osaka Red Cross

Hospital, 5-30 Fudegasakicho, Tennoji-ku,

Osaka 543-8555, Japan

e-mail: masayuki@akimoto3.com repeated practice using the two-hole method. The drilling of holes enabled deep and effortless impaling of the nucleus. Although there was no significant difference in the $\mathrm{CDE}$ among the techniques, there was a significant difference in the UST $(P<0.05)$.

Conclusion The two-hole method enabled surgeonsin-training to acquire standard phaco-chop skills more efficiently. However, further studies with higher statistical power will be needed to validate these findings. Additionally, a variation of this technique, the four-hole method, is applicable even for experienced surgeons in cases of a hardened nucleus.

Keywords Phaco-chop - Nucelofractis · Two-hole · Cataracts

\section{Introduction}

A successful nuclear fracture is a crucial step in the phacoemulsification technique. Divide-and-conquer, presented by Gimbel in 1991 [1], is a standard and widely accepted technique used by cataract surgeons. Phaco-chop, developed by Nagahara in 1993 [2], is considered one of the most effective methods for nuclear fracturing and requires less time for ultrasound sculpting [3-5]. Some variants, such as the twodimensional horizontal chop or three-dimensional vertical chop, have been developed and accepted for 
use. The vertical chop method enables deep nuclear penetration and is particularly effective for the management of a hardened nucleus. Many modified techniques for hard cataracts have been described wherein the central part of the nucleus is drilled (or dug) to facilitate its disassembly [6-8].

Although frequently compared with the divide-andconquer method, phaco-chop reduces the ultrasound energy required for nucleofractis. However, young surgeons need extensive efforts and training to shift from the divide-and-conquer technique to phaco-chop successfully. Usually, phaco-chop is taught to new surgeons partially, for example, only in the second and third chopping.

The correct placement of the chopper is one of the difficult maneuvers in phaco-chop. Surgeons-in-training tend to lacerate the capsulorhexis when they attempt to divide the nucleus from the periphery. If the pupil size is small, such as in patients with intraoperative floppy iris syndrome (IFIS), the capsulorhexis tends to be inadequate. This increases the risk of lacerating the capsulorhexis or pupillary margin by the chopper.

In cases where there is an excessively soft or a hard nucleus, embedding both a phaco-tip and a chopper to a depth sufficient to sandwich and chop the lens may also be difficult, especially in the first chop.

Young surgeons must acquire different left-hand movements to shift from the divide-and-conquer technique to phaco-chop. Herein, we report our new technique, which would enable effortless mastery of the phaco-chop technique. The basis of our technique is the generation of a new lens equator inside the capsulorhexis by drilling two holes. Drilling two holes adjacent to the capsulorhexis mimics a new lens equator; the lens appears smaller, enabling surgeonsin-training to master the phaco-chop more safely and easily without pushing the lens posteriorly.

\section{Methods}

Three surgeons in their first year of training (fellows $\mathrm{A}, \mathrm{B}$, and $\mathrm{C}$ ) performed the phaco-chop technique. Before learning this technique, two attending surgeons had previously experienced grooving the nucleus, whereas the third surgeon had not. That is, the third surgeon directly acquired knowledge of the phaco- chop technique after repeatedly practicing the twohole method.

We compared the cumulative dissipated energy (CDE) and case ultrasound time (UST) with three phacoemulsification techniques (divide-and-conquer, two-hole method, and phaco-chop) among the three young surgeons. We collected data of their first 8 cases and analyzed a total of 64 cases: 16, divide-andconquer; 24, two-hole method; 24, phaco-chop.

\section{Surgical technique}

In this study, the Constellation Vision System (Alcon Laboratories, Inc., Fort Worth, TX, USA) was used. Phacoemulsification was performed with a $45^{\circ} \mathrm{Kel}-$ man Mini tip under the flow mode. For the two-hole technique, the phaco-tip was buried into the nucleus about 3/4 depth of the lens adjacent to the capsulorhexis; this step was repeated after rotating the nucleus by $180^{\circ}$ to make two holes on the nucleus. We used sleeve irrigation port as a reference for the depth. To avoid damaging the anterior capsule, the phaco-tip was carefully placed inside the capsulorhexis and then pulled until the phaco-tip stood as vertically as possible. Phacoemulsification power was maintained low at $20 \%$ to reduce the risk of damage to the posterior capsule in the early stages of the surgery. Power can be increased as the surgeons-in-training get used to this technique. Other settings for drilling holes were vacuum $220-250 \mathrm{mmHg}$, aspiration flow rate $30 \mathrm{~mL} / \mathrm{min}$, phaco power $20-40 \%$, and infusion bottle height $85 \mathrm{cmH}_{2} \mathrm{O}$.

Before drilling the two holes on the nucleus adjacent to the capsulorhexis, the lens nucleus was separated from the capsule to enable its smooth rotation in the capsular bag. Hydrodissection and removal of the cortex from the periphery were performed sufficiently beforehand.

The phaco-tip was inserted into one of the holes. Thereafter, the chopper was inserted deeply into the other hole (Fig. 1a, b). The two instruments were moved back and forth relative to each other until the nucleus cracked into two separate pieces. The supplementary videos (Video 1,2) show a shortened version of the procedure used in this study.

After drilling the two holes on the nucleus adjacent to the capsulorhexis, nucleofractis and quadrant removal were performed under the following settings: phaco power $0 \%$, torsional power $70 \%$, vacuum 380 
Fig. 1 a Top view of the chopper and the phaco-tip being buried into the two holes (yellow arrows) adjacent to the capsulorhexis. b Side view of the placement of the chopper and phaco-tip as described in a. c and $\mathbf{d}$ Two holes (orange arrows), additional to the original holes (yellow arrows), are drilled adjacent to the capsulorhexis. Drilling four holes enables effective nuclear fracturing in cases with a hardened nucleus
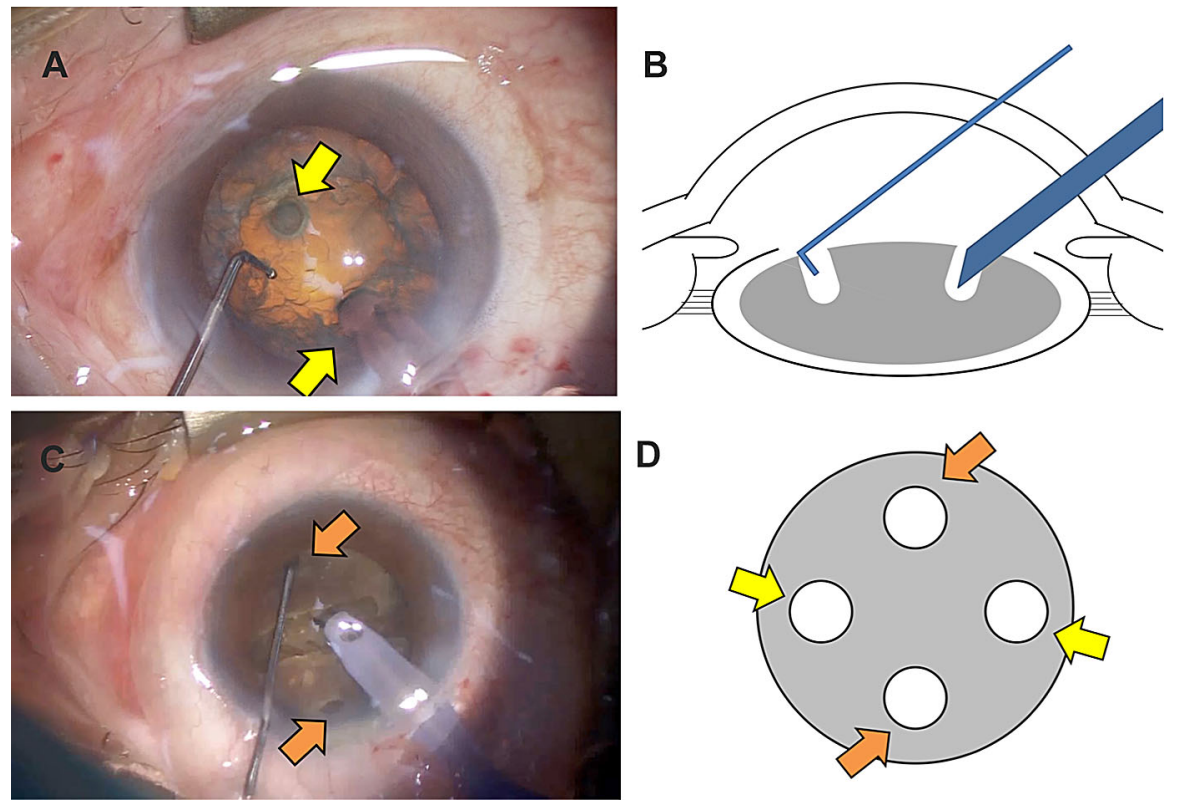

$\mathrm{mmHg}$, aspiration flow rate $40 \mathrm{~mL} / \mathrm{min}$, and infusion bottle height $90 \mathrm{cmH}_{2} \mathrm{O}$.

\section{Results}

The young practicing surgeons could eventually perform the standard phaco-chop, with the two-hole method, more easily and successfully.

The drilling of holes enabled deep and effortless impaling of the nucleus.

Almost all these maneuvers were performed inside the capsulorhexis; therefore, the risk of laceration was minimized. Despite the small pupil size or presence of IFIS, the first-year fellows successfully completed the procedure. In a few cases, we found round continuous notches of the anterior capsulorhexis in the two-hole group because of drilling over the anterior capsule. We did not count this as a complication because the continuity and the integrity of capsulorhexis were not altered. Among the 64 cases, there were four intraoperative complications: one small zonular dehiscence each in the divide-and-conquer group and in the twohole group, one posterior capsule rupture with vitreous loss in the two-hole group, and one radial capsulorhexis tear in the phaco-chop group. The case with posterior capsule rupture was excluded during the calculation of the average, and the next consecutive case was considered in place of the excluded case. There were no early postoperative complications.

Figure 2a shows the analysis of CDE data for each technique. CDE was $11.4 \%$-sec for divide-and-conquer, $8.5 \%$-sec for the two-hole technique, and 7.2\%sec for phaco-chop. Although the comparison between each group showed a significant difference between the divide-and-conquer and phaco-chop techniques $(P=0.0371)$, a three-group comparison with the nonparametric Kruskal-Wallis test did not reveal any significant differences $(P=0.0538)$.

On the contrary, significant differences were seen in UST $(P=0.0008$; Fig. $2 b)$ among the three techniques. UST was $91.4 \mathrm{~s}$ for divide-and-conquer, $55.8 \mathrm{~s}$ for the two-hole technique, and $44.5 \mathrm{~s}$ for phaco-chop. Significant differences were also observed in twogroup comparisons: Divide-and-conquer vs. two-hole $(P=0.0219)$; divide-and-conquer vs. phaco-chop $(P=0.0004) ; \quad$ and two-hole vs. phaco-chop $(P=0.0477)$.

\section{Discussion}

To minimize tissue damage and complications, the phaco power must be reduced and used efficiently. The phaco-chop technique is one of the most effective methods for nuclear fracturing and requires less time for ultrasound sculpting. In this study, we aimed for 


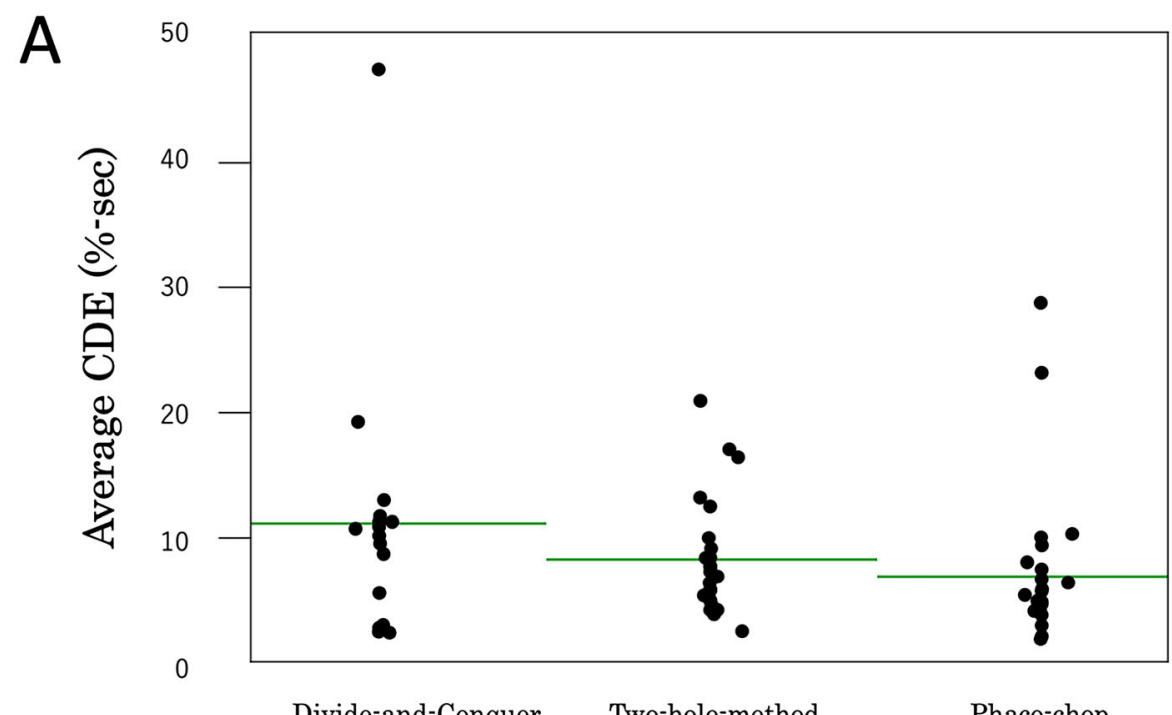

Divide-and-Conquer Two-hole-method Phaco-chop

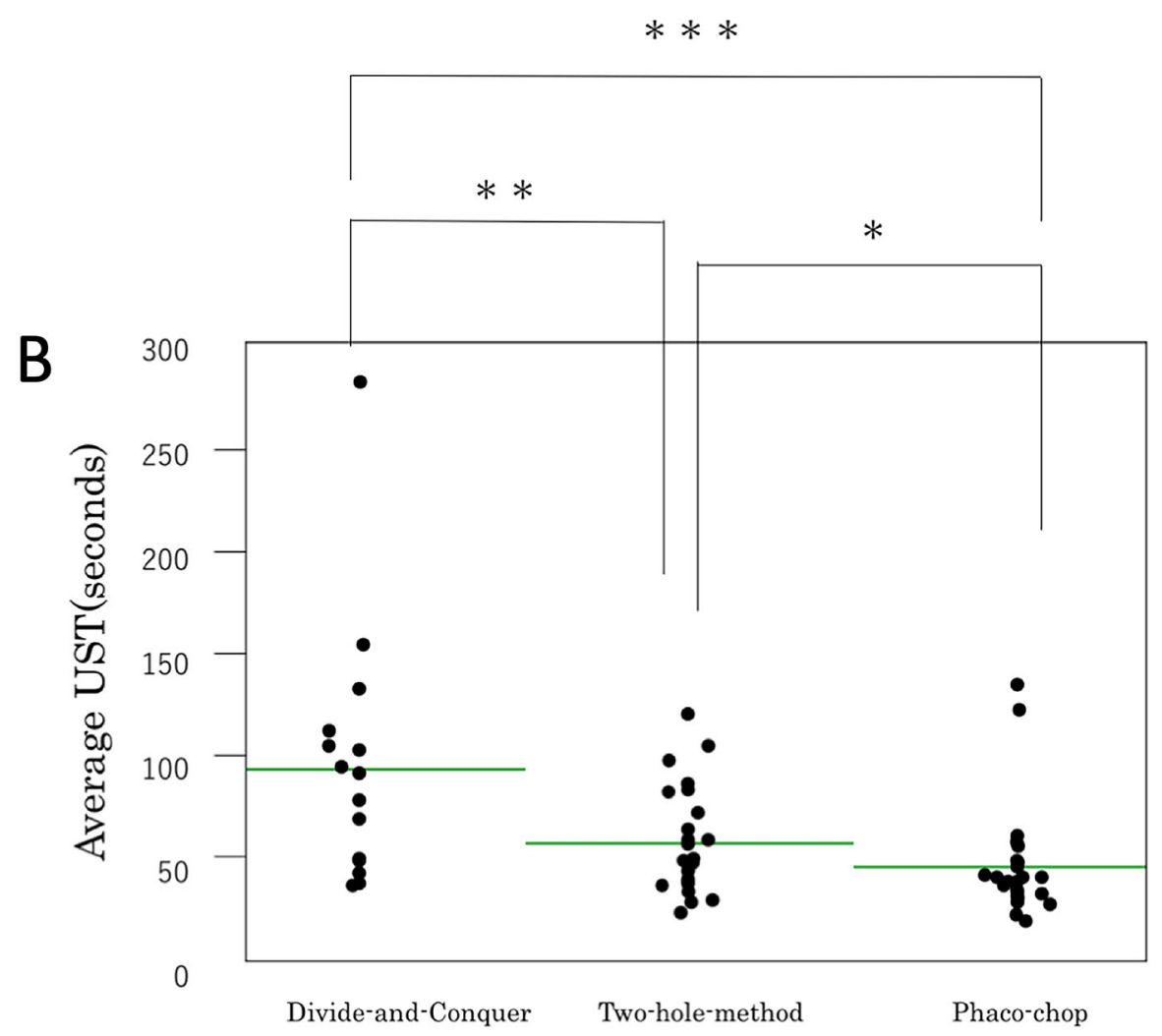

Fig. 2 a Comparison of cumulative dissipated energy among the three techniques. A significant difference was not observed among the groups $(P=0.0538)$ with the non-parametric Kruskal-Wallis test. b Comparison of the loss of ultrasound time among the three techniques. There was a significant difference among the groups $(P=0.0008)$ with the nonparametric Kruskal-Wallis test. **Divide-and-conquer vs. two-hole $(P=0.0219)$. ***divide-and-conquer vs. phaco-chop $(P=0.0004)$ *two-hole vs. phaco-chop $(P=0.0477)$ 
the surgeons-in-training to master the phaco-chop technique steadily and quickly. While focusing on enabling mastery of the first chopping, we envisioned this technique of drilling two holes adjacent to the capsulorhexis that would mimic a new lens equator for easier first chopping. Using this approach, young surgeons who are attempting the technique for the first time can efficiently remove the initial segment.

There are only a few studies about the different phacoemulsification techniques efficiency among surgeons-in-training. Wong et al. reported an average UST with phaco-chop of $72 \mathrm{~s}$, which was less than the average UST with divide-and-conquer (124 s) [4]. Although the equipment used and the conditions were different, our study also shows an advantage of a shorter UST with the phaco-chop technique.

Coppola et al. compared the efficiency of surgeonsin-training when performing the divide-and-conquer and stop-and-chop techniques. They reported that even for surgeons-in-training, the stop-and-chop technique was more efficient for advanced cataracts and encouraged a switch from divide-and-conquer to stopand-chop [9].

Gross et al. compared the efficiency of nuclear disassembly among resident surgeons while using the divide-and-conquer technique and the pop-and-chop technique, [10] which was described by Pandit and Oetting in 2003 [11]. The pop-and-chop technique enables an easy first crack after partial extracapsular prolapse of the nucleus prior to the initial chop. They reported a CDE of $15.9 \%$-sec with divide-and-conquer and $8.6 \%$-sec with pop-and-chop. The average surgical time was $31 \mathrm{~min}$ and $17.8 \mathrm{~min}$, respectively. Although pop-and-chop is a more time- and energyefficient technique than divide-and-conquer for nucleofractis in the case of novice resident surgeons, the first chop is performed on the bag space. In contrast, our two-hole technique is performed in the bag space, which is the same as the standard and other advanced techniques. Regarding efficiency, although the equipment and the conditions may be different, our twohole technique shows similar CDE to that of the popand-chop technique, and our surgeons-in-training could migrate the techniques to the standard in-thebag phaco-chop technique effortlessly.

This method can also be implemented by experienced surgeons. Drilling four holes adjacent to the capsulorhexis enables effective nuclear fracturing in cases with a hardened nucleus (Fig. 1c, d). First, through two of the four holes drilled in opposite angles, the nucleus is split into two pieces according to the conventional phaco-chop technique. Thereafter, using the other two holes, each hemi-nucleus is chopped into two smaller pieces, resulting in the nucleus splitting into four pieces. This variation enables more effective nucleus fracturing than the conventional methods of grooving denser cataracts.

In cases of a temporal approach for the left eye, surgeons may rotate the surgical microscope and position themselves at the temporal side of the patient. However, drilling holes enables a regular superior approach more easily without requiring a change in their sitting positions.

Thus, these holes adjacent to the capsulorhexis, which mimic a smaller lens equator, enable more efficient phaco-chopping not only for surgeons-intraining but also for experienced surgeons.

Although we observed a difference in the UST, the sample size and power of this study were insufficient for conclusive results. Difficulty in the preparation of cataracts of the same type and density among these three surgeons might have reduced the accuracy of the study by introducing sample bias. Surgeons should have improved their skills after every case. For accurate analysis, all three surgeons should have started learning these three techniques simultaneously. Therefore, further studies with a larger sample size are needed to validate our findings that our technique reduces both energy levels and UST when compared with conventional techniques like the divide-andconquer method.

\section{Conclusion}

This study aimed to enable mastery of the phaco-chop technique by surgeons-in-training steadily and quickly. Although no significant difference was found among the techniques in the $\mathrm{CDE}$, there was a significant difference in the UST $(P<0.05)$.

Our results suggest that the two-hole method may improve efficiency among surgery-residents-in-training. Owing to flaws in the design of the study, possible bias, and lack of statistical power, further studies with a larger sample size may be needed to examine the use of the two-hole method in nucleofractis techniques. 
Acknowledgements We would like to thank Editage [http:// www.editage.com] for editing and reviewing this manuscript for English language.

Authors' contribution NM wrote this manuscript. MA designed the study. All authors discussed the procedure, performed the surgeries, and examined patients.

\section{Declarations}

Conflict of interest M. A. is a consultant at Kowa Co. Ltd. No conflicts of interest exist for the other authors.

Ethical approval This study has been approved by the institutional ethics committee and has been performed in accordance with the ethical standards as laid down in the 1964 Declaration of Helsinki and its later amendments or comparable ethical standards.

Consent to participate Informed consent was obtained from all patients included in the study.

Consent for publication Patients have consented to the usage of pictures and videos in this paper and the publication of this study.

Open Access This article is licensed under a Creative Commons Attribution 4.0 International License, which permits use, sharing, adaptation, distribution and reproduction in any medium or format, as long as you give appropriate credit to the original author(s) and the source, provide a link to the Creative Commons licence, and indicate if changes were made. The images or other third party material in this article are included in the article's Creative Commons licence, unless indicated otherwise in a credit line to the material. If material is not included in the article's Creative Commons licence and your intended use is not permitted by statutory regulation or exceeds the permitted use, you will need to obtain permission directly from the copyright holder. To view a copy of this licence, visit http://creativecommons.org/licenses/by/4.0/.

\section{References}

1. Gimbel HV (1991) Divide and conquer nucleofractis phacoemulsification: development and variations. J Cataract
Refract Surg 17:281-291. https://doi.org/10.1016/S08863350(13)80824-3

2. K. Nagahara (1993) "Phaco Chop" film presented at the 3rd American-International Congress on Cataract, IOL and Refractive Surgery, Seattle, Washington, USA, June 1993

3. DeBry P, Olson RJ, Crandall AS (1998) Comparison of energy required for phaco-chop and divide and conquer phacoemulsification. J Cataract Refract Surg 24:689-692. https://doi.org/10.1016/S0886-3350(98)80267-8

4. Wong T, Hingorani M, Lee V (2000) Phacoemulsification time and power requirements in phaco chop and divide and conquer nucleofractis techniques. J Cataract Refract Surg 26:1374-1378. https://doi.org/10.1016/S0886-3350(00)00 $538-1$

5. Koch PS, Katzen LE (1994) Stop and chop phacoemulsification. J Cataract Refract Surg 20:566-570. https://doi.org/ 10.1016/S0886-3350(13)80239-8

6. Hwang HS, Kim EC, Kim MS (2010) Drill-and-crack technique for nuclear disassembly of hard nucleus. J Cataract Refract Surg 36:1627-1630. https://doi.org/10. 1016/j.jcrs.2010.08.003

7. Kim DY, Jang JH (2012) Drill and chop: modified vertical chop technique for hard cataract. Ophthalm Surg Lasers Imaging 43:169-172. https://doi.org/10.3928/1542887720111222-01

8. Elmazar HM (2018) Dig and Chop Modified Nuclear Disassembly Technique for Hard Cataract. J Ophthalmol Vis Sci 3:102

9. Coppola M, Marchese A, Rabio A, Cicinelli MV, Knutsson KA (2019) Comparison of two popular nuclear disassembly techniques for cataract surgeons in training: divide and conquer versus stop and chop. Int Ophthalmol 39:207-2102. https://doi.org/10.1007/s10792-018-1046-4

10. Gross FJ, Garcia-Zalisnak DE, Bovee CE, Strawn JD (2016) A comparison of pop and chop to divide and conquer in resident cataract surgery. Clin Ophthalmol 10:1847-1851. https://doi.org/10.2147/OPTH.S115840

11. Pandit RT, Oetting TA (2003) Pop-and-chop nucleofractis. J Cataract Refract Surg 29:2054-2056. https://doi.org/10. 1016/S0886-3350(03)00339-0

Publisher's Note Springer Nature remains neutral with regard to jurisdictional claims in published maps and institutional affiliations. 